\title{
STANDARDIZATION OF THE LABORATORY RESEARCH PROTOCOL AND \\ ANATOMOPATHOLOGICAL DIAGNOSIS OF BREAST GRANULOMATOUS INJURIES
}

Daniella Serafin Couto Vieira', Bráulio Leal Fernandes', Érica Elaine Traebert Simezo, Amanda Amaro Pereira, Mara Scheffer ${ }^{1}$

${ }^{1}$ Universidade Federal de Santa Catarina - Florianópolis (SC), Brazil.

Introduction: Granulomatous breast lesions are multifactorial conditions, with clinical, mammographic, and ultrasound findings like those observed in cases of breast carcinoma. Histological evaluation can present key characteristics to define the lesion pattern. Although this entity is rarely reported in the literature, it is associated with inflammatory conditions such as Ductal Ectasia and foreign body reaction. However, it can also be associated with agents such as bacteria, fungi, and parasites. Furthermore, idiopathic causes and exclusion diagnosis, such as sarcoidosis, can be included in the etiology of the process. Objectives: To establish a correlation between the anatomopathological diagnosis and the laboratory investigation by culture for breast granulomatous lesions diagnosis, with validation of the sample's analysis protocol. Methods: Samples were selected from 17 women treated at the Mastology Service of a Public Hospital in Brazil, with a history and physical examination that raised suspicion of breast granulomatous lesion and they had previous clinical indication of breast core biopsy. The collection of samples was guided by ultrasonography (USG). In turn, they were stored in a blood culture flask (BD BACTECTM) to perform culture tests by automation (VITEK2), bacterioscopy and fungi exams, and acid resistant bacillus (ARB) tests at the Laboratory of Clinical Analyses. Simultaneously, core biopsy samples, fixed in $10 \%$ buffered formaldehyde, were sent to a Laboratory of Pathological Anatomy, for a morphological evaluation and research of ARB, fungi, and other bacteria, using the Ziehl-Nielsen, Grocott, PAS and GRAM histochemical methods.

Results: Of the 17 samples, 11 had a chronic inflammatory response pattern with a non-lobulocentric granulomatous reaction component and one of them had a lymphocytic mastitis pattern. The five patients with morphological pattern of lobulocentric granulomatous mastitis presented positive culture, four for Corynebacterium kroppenstedtil, and one for Staphylococcus hominis. Sensitivity, Specificity, Positive Predictive Value and Negative Predictive Value in this sample was $100 \%$. Conclusion: The clinical and radiological aspects can bring difficulties that obscure the diagnostic and etiological interpretation of granulomatous lesions. Thus, the morphological details observed in the anatomical pathological examination and the use of the laboratory investigation protocol with standardization of histochemical reactions associated with the use of tools for microbiological diagnosis show increased sensitivity and specificity for the detection of specific etiologic agents in granulomatous mastitis. 